\title{
Incorrect formula for determining the volume of a muscle in vivo
}

\author{
Hope Gangata
}

Accepted: 23 April 2009/Published online: 10 May 2009

(C) Springer-Verlag 2009

Esformes et al. (2002) wrote an excellent paper describing the high validity and reliability of a technique using ultrasonography to determine the volume of the tibialis anterior muscle. Ultrasonography has advantages over MRI in estimating the volume of muscles in vivo, in that it is far cheaper and does not produce ionising radiation. However the formula presented for calculating volume, as Equation 1, appears to be incorrect. The volume of a solid is generally found by multiplying the cross-section area by the length. In their paper, the volume was a summation of ten volumes. The formula omitted the lengths of the volumes. The formula presented in their article was

$V=\frac{1}{3} \times[a+\sqrt{ }(a b+b)]$

where $a$ and $b$ are the adjacent cross-section areas. The formula should instead be

$V=\frac{1}{3} \times[a+\sqrt{ }(a b+b)] \times t$ where $t$ is the inter-scan distance (length) between the adjacent areas. Esformes et al. (2002), appear to have used the correct formula for the calculation of muscle volume and the scientific merits of their paper are thus preserved. The adjusted volume formula can be used to estimate the volume of other muscles to monitor hypertrophy and muscle wasting. The volume of a muscle is also essential for calculating the physiological cross-section area, which is correlated to muscle power. The formula has been under use in ultrasonographic studies and has the potential to be used to measure the volume organs and tumours that are not covered by bones.

\section{References}

Esformes JI, Narici MV, Maganaris CN (2002) Measurement of human muscle volume using ultrasonography. Eur J Appl Physiol 87(1):90-92. doi:10.1007/s00421-002-0592-6

H. Gangata $(\bowtie)$

University of Cape Town, Cape Town, South Africa

e-mail: hopegata@yahoo.co.uk 